\title{
Análise Visual utilizando Projeções Multidimensionais
}

\author{
Tácito T. de A. T. Neves ${ }^{1}$ \\ Samuel G. Fadel ${ }^{2}$ \\ Danilo B. Coimbra ${ }^{2}$ \\ Fernando V. Paulovich ${ }^{2}$
}

Data de submissão: 16.06 .2015

Data de aceitação: 11.11 .2015

\begin{abstract}
Resumo: As técnicas de projeção multidimensional, ou também conhecidas como técnicas de redução de dimensionalidade, vêm despertando grande interesse da comunidade de visualização e análise de dados por possibilitarem que a capacidade visual humana seja empregada na exploração e interpretação de relações de similaridade e vizinhança. Atualmente, essa é uma das ferramentas de visualização mais empregadas para a exploração de dados multidimensionais e sua aplicação se estende por vários domínios como, por exemplo, análise de dados de sensores e biosensores e exploração de coleções de imagens e músicas. Este artigo apresenta uma visão geral de técnicas clássicas e do estado-da-arte para projeção multidimensional, algumas ferramentas disponíveis, bem como exemplos de aplicações, buscando evidenciar como as projeções podem assistir na exploração e entendimento de diferentes conjuntos de dados.
\end{abstract}

\begin{abstract}
Multidimensional projection techniques, also known as dimensionality reduction techniques, have attracted great interest among the visualization and data analysis community due to its capacity of enabling the human visual ability to be employed on the interpretation and analysis of similarity and neighborhood relationships. Currently, projections are one of the most popular visualization tools for the exploration of multidimensional datasets. Its application goes from the analysis of sensors and biosensors data to the browsing of images and music collections. This paper presents a survey of classical and state-of-the-art multidimensional projection techniques, some available tools, and examples of applications aiming at showing how projections can assist on the exploratory analysis and understanding of different data sets.
\end{abstract}

\footnotetext{
${ }^{1}$ Universidade Federal de Viçosa, Rio Paranaíba - MG

tacito.neves@ufv.br

${ }^{2}$ Universidade de São Paulo, São Carlos - SP

samuel.fadel@usp.br, \{danilobc, paulovic\}@icmc.usp.br
} 


\section{Introdução}

Com a diminuição do custo e a melhoria das tecnologias para armazenamento, distribuição e recuperação de dados, a quantidade de informação produzida, ou disseminada, tem crescido substancialmente tanto em volume quanto em complexidade. Apesar desse aumento parecer positivo, excesso de informação pode resultar em um efeito conhecido como "sobrecarga de informação". A sobrecarga de informação ocorre quando o volume de informação é tão grande que uma pessoa não é capaz de localizar e fazer uso do que é necessário [15].

Apesar de muita informação não relevante poder ser filtrada, a geração de informação útil ainda continua sendo muito maior do que a capacidade das ferramentas de interpretação vigentes, tornando necessário o desenvolvimento de métodos e ferramentas capazes de sintetizar esse aglomerado de dados para que seja possível interpretá-lo, apresentando-o de forma simples e amigável.

Dentre as áreas que estudam métodos para apoiar a interpretação de tal informação, a área de Visualização de Informações tem despertado grande interesse por permitir que a capacidade visual humana seja empregada no processo exploratório, acelerando a extração de conhecimento. Diferentes técnicas de visualização têm sido propostas. A maioria mapeia cada item de dado em um elemento gráfico correspondente, que pode ser um pixel, uma linha, um ícone ou outro marcador. De acordo com Grinstein et al. [23], alguns exemplos tradicionais seriam: scatterplots 2D e 3D [16], Matrizes de scatterplots [2], Table lens [56], técnicas Iconográficas [14, 54, 8, 58], Empilhamento Dimensional [38], Coordenadas Paralelas [27, 29, 28], Técnicas Orientadas a Pixel [33, 34, 32], Segmentos de Círculo [4], RadViz [25], Grand Tour [6], Mapas Auto-organizáveis de Kohonen [36], etc, sendo que muitas dessas são similares ou estão relacionadas. Um estudo sobre algumas dessas técnicas foi elaborado por Oliveira e Levkowitz [43].

Embora tais técnicas possam ser empregadas com sucesso em pequenos conjuntos de dados, para conjuntos maiores com alta dimensionalidade elas tendem a apresentar problemas, não conseguindo identificar satisfatoriamente grupos de dados correlacionados, determinar elementos espúrios ou tendências. Uma abordagem alternativa a essas técnicas tradicionais, que tem sido aplicada com sucesso na visualização de dados com alta dimensionalidade, são as técnicas de Projeção Multidimensional [65]. Uma técnica de projeção multidimensional mapeia as instâncias de dados em um espaço uni-, bi- ou tri-dimensional, preservando alguma informação sobre as relações de distância ou similaridade entre elas de forma a revelar o máximo possível às estruturas existentes. Tipicamente, o resultado de uma projeção multidimensional é um conjunto de pontos no plano, numa reta ou num volume, cada elemento representando uma instância de dados. Numa projeção de boa qualidade, pontos projetados próximos indicam instâncias similares, e os distantes, as não correlacionadas de acordo com a alguma medida de similaridade. Dentre as vantagens desse tipo de abordagem, destacam-se 
a possibilidade identificar e extrair padrões que não são detectáveis por métodos clássicos de análise e fornecer ao usuário maior confiança nos resultados obtidos se comparado com outras técnicas [61].

Assim, projeções multidimensionais tem se mostrado como uma solução efetiva à interpretação de conjuntos multidimensionais de dados, dando apoio e suporte à rápida extração de informação relevante desse tipo de dado, configurando, portanto, um mecanismo que pode efetivamente contribuir para diminuir os problemas associados à sobrecarga de informação.

As próximas seções estão organizadas da seguinte maneira: a seção 2 apresenta a fundamentação teórica e os trabalhos relacionados ao estudo, bem como as técnicas de projeção multidimensionais abordadas nesta revisão; na seção 3 são apresentadas algumas aplicações existentes que utilizam técnicas de projeção multidimensional para realizar análise exploratória sobre diversos tipos de dados; na seção 4, algumas ferramentas disponíveis são demonstradas; e na seção 5 , as conclusões gerais e possíveis contribuições futuras são relatadas, além de tendências da área.

\section{Técnicas de Projeção}

De forma geral, as técnicas de projeção multidimensional são uma importante ferramenta analítica devido à sua capacidade de revelar estruturas de similaridade e vizinhança em espaços multidimensionais. Análises de campos vetoriais [18], mineração visual de texto [13, 71, 3], e análise de sensores e biossensores [46, 53, 47] são apenas alguns exemplos de aplicações nas quais projeções vem sendo empregadas com sucesso.

Matematicamente, sejam $\mathcal{X}=\left\{\mathbf{x}_{1}, \mathbf{x}_{2}, \ldots, \mathbf{x}_{n}\right\}$ um conjunto de dados contendo $n$ tuplas tais que $\mathbf{x}_{i} \in \mathbb{R}^{p}$ representam $n$ observações com $p>3$ variáveis e $\delta: \mathbb{R}^{p} \times$ $\mathbb{R}^{p} \rightarrow \mathbb{R}_{0}^{+}$uma função de dissimilaridade entre duas instâncias [17]. Seja também $\mathcal{Y}=$ $\left\{\mathbf{y}_{1}, \mathbf{y}_{2}, \ldots, \mathbf{y}_{n}\right\}$ um conjunto de $n$ tuplas tais que $\mathbf{y}_{i} \in \mathbb{R}^{q}(q \in\{1,2,3\})$. Uma técnica de projeção multidimensional é um método que encontra uma função injetiva $f: \mathcal{X} \rightarrow \mathcal{Y}$ que busca preservar em $\mathcal{Y}$ alguma característica original dos dados, em geral as relações de similaridade ou vizinhança. Os conjuntos $\mathcal{X}$ e $\mathcal{Y}$ serão representados também como $X$ e $Y$, respectivamente, quando uma representação matricial for mais adequada. Nesta representação matricial, considera-se que cada linha $i$ da matriz $X$ (ou $Y$ ) contém as coordenadas de $\mathbf{x}_{i}$ (ou $\left.\mathbf{y}_{i}\right)$.

Uma das taxonomias mais aceitas para a classificação das técnicas de projeção divide as mesmas em dois grandes grupos, técnicas globais e técnicas locais [20]. Técnicas globais buscam aproximar $\delta\left(\mathbf{x}_{i}, \mathbf{x}_{j}\right)$ de $\left\|\mathbf{y}_{i}-\mathbf{y}_{j}\right\|$, para todo $i \neq j$, enquanto que técnicas locais buscam preservar as distâncias considerando pequenas vizinhanças [20]. De forma geral, se o 
objetivo é preservar a média das distâncias, técnicas globais são a melhor escolha. Caso o objetivo seja a preservação das vizinhanças, é preferível utilizar técnicas locais. Nas subseções seguintes, algumas técnicas desses dois grupos são apresentadas.

\subsection{Técnicas Globais}

Dentre as primeiras técnicas globais desenvolvidas, as técnicas denominadas Multidimensional Scaling (MDS) foram as que atingiram maior popularidade [17]. Dentre os métodos de MDS mais conhecidos temos o Classical Scaling [67] e o Least Squares Scaling. No Classical Scaling, busca-se uma transformação linear que mapeie $\mathcal{X}$ num espaço Euclidiano onde as dissimilaridades $\delta\left(\mathbf{x}_{i}, \mathbf{x}_{j}\right)$ se aproximam das distâncias $\left\|\mathbf{y}_{i}-\mathbf{y}_{j}\right\|$. Essa transformação é encontrada fazendo a decomposição espectral da matriz de dissimilaridades. Quando as dissimilaridades são Euclidianas, existem garantias para se encontrar um espaço onde elas podem ser representadas sem perdas, porém, na prática, são necessárias mais de 3 dimensões. Já no Least Squares Scaling, uma configuração dos pontos é buscada de forma que $\left\|\mathbf{y}_{i}-\mathbf{y}_{j}\right\| \approx g\left(\delta\left(\mathbf{x}_{i}, \mathbf{x}_{j}\right)\right)$ por meio da minimização de uma função de perda, sendo $g$ uma função de transformação.

Um exemplo de Least Squares Scaling é a técnica Sammon's Mapping [60]. Nessa técnica, a seguinte função de perda é minimizada:

$$
S_{1}=\frac{1}{\sum_{i<j} \delta\left(\mathbf{x}_{i}, \mathbf{x}_{j}\right)} \sum_{i<j} \frac{\left(\left\|\mathbf{y}_{i}-\mathbf{y}_{j}\right\|-\delta\left(\mathbf{x}_{i}, \mathbf{x}_{j}\right)\right)^{2}}{\delta\left(\mathbf{x}_{i}, \mathbf{x}_{j}\right)} .
$$

Como essa função é ponderada por $\delta\left(\mathbf{x}_{i}, \mathbf{x}_{j}\right)^{-1}$, as pequenas dissimilaridades terão maior peso que as grandes, tornando o Sammon's Mapping capaz de desdobrar dados de manifolds de alta dimensão. Esta opção é preferida às ponderações drásticas como $\delta\left(\mathbf{x}_{i}, \mathbf{x}_{j}\right)^{-2}$ que não auxiliam a atingir um balanço entre preservação local e global de estruturas, particularmente quando se está trabalhando dentro de pequenas vizinhanças [39].

Na Sammon's Mapping, as distâncias $d\left(\mathbf{y}_{i}, \mathbf{y}_{j}\right)$ e as dissimilaridades $\delta\left(\mathbf{x}_{i}, \mathbf{x}_{j}\right)$ normalmente são Euclidianas, sendo que para minimização da função de perda é usado um método iterativo não-linear que emprega o gradiente dessa função para se encontrar um mínimo local [52]. A $t$-ésima iteração desse método é definida pela equação abaixo:

$$
y_{i j}(t+1)=y_{i j}(t)-M F \times \Delta_{p q}(t),
$$

onde $y_{i j}$ denota a coordenada $j$ do ponto $\mathbf{y}_{i}$ e

$$
\Delta_{i j}(t)=\frac{\partial S_{1}(t)}{\partial y_{i j}(t)} /\left|\frac{\partial^{2} S_{1}(t)}{\partial y_{i j}^{2}(t)}\right|,
$$


onde $0<M F \leq 1$ é um "fator mágico" que serve para otimizar a convergência do algoritmo, sendo determinado originalmente como $0.3 \leq M F \leq 0.4$. Para acelerar a convergência a um mínimo, Chang e Lee [11] usaram um método de relaxamento heurístico para determinar o $M F$ e Niemann e Weiss [42] usaram um tamanho ótimo de passo calculado a cada iteração ao invés do $M F$.

Os métodos MDS originais são muito precisos em termos de preservação da distância global, mas são computacionalmente caros. Como forma de melhorar essa complexidade, mas ainda buscando uma boa qualidade em termos de preservação da distância, algumas estratégias foram propostas: Landmarks MDS (LMDS) [63], Pivot MDS [9] e a abordagem de Pekalska [52] que projetam um subconjunto pequeno de instâncias e por meio de interpolação encontram o mapeamento final.

Uma técnica recente que busca uma maior velocidade no processo de projeção é a Part-Linear Multidimensional Projection (PLMP) [51]. Na PLMP, inicialmente uma projeção $\mathcal{Y}^{\prime} \subset \mathbb{R}^{q}$ é construída para um subconjunto de instâncias representativas $X^{\prime} \subset \mathbb{R}^{p}$ e essa informação é usada para a construção de uma transformação linear $\Phi: \mathbb{R}^{p} \rightarrow \mathbb{R}^{q}$ para o posicionamento das outras instâncias não projetadas. A transformação $\Phi$ pode ser aproximada de forma eficiente resolvendo um sistema linear $\Phi X^{\prime}=Y^{\prime}$ por meio de mínimos quadrados. Uma vez que $\Phi$ for determinada, a projeção do conjunto de dados passar a ser a transformação de cada $\mathbf{x}_{i} \notin \mathcal{X}^{\prime}$ por $\Phi$, uma operação pouco custosa.

Na PLMP, a qualidade do mapeamento linear depende basicamente da seleção das instâncias representativas e de posicionamento delas no espaço visual. A possibilidade de mudança interativa do posicionamento das instâncias representativas é uma das características mais interessantes da PLMP, tornando-a um mecanismo verdadeiramente interativo para análise visual de dados. Entre as limitações da técnica, está a necessidade de que o número de instâncias representativas seja maior do que o número de dimensões dos dados, o que pode dificultar o uso da técnica em situações nas quais os dados estão em um espaço dimensional muito alto.

Uma abordagem diferente é apresentada na técnica Glimmer [26]. Glimmer é um algoritmo MDS baseado em força que utiliza um arcabouço hierárquico recursivo para aumentar a precisão e reduzir o custo computacional. Em sua estratégia multi-nível, Glimmer tem como nível mais alto o conjunto inteiro dos dados. Os níveis mais baixos são subconjuntos aninhados, reduzidos por um fator fixo. O fator de redução de um nível para o outro é igual a 8 , até que o número de instâncias seja menor do que 1000 .

Em cada nível da hierarquia, a técnica faz uso de três operadores sobre as instâncias do subconjunto correspondente: restriction, obtém os subconjuntos para construir a hierarquia; relaxation, operador que reduz o erro da aproximação das distâncias no espaço visual até um valor específico; e, interpolation, passa o resultado do operador relaxation para o próximo 
nível da hierarquia.

O diferencial apresentado pela Glimmer é a possibilidade de implementação em GPU, melhorando o tempo de processamento. O uso da GPU, quando comparado com a execução em CPU, não afeta a convergência e a precisão, apenas diminui o tempo de processamento. A Glimmer de forma geral busca minimizar uma função de stress, que calcula as diferenças entre as distâncias no espaço original e no espaço transformado. Porém, ao invés de minimizar as diferenças par-a-par, faz esse processo empregando amostragem, minimizando

$$
\text { sparsestress }=\sqrt{\frac{\sum_{i} \sum_{j \in \operatorname{Near}(i) \cup \operatorname{Random}(i)}\left(d_{i j}-\delta_{i j}\right)^{2}}{\sum_{i} \sum_{j \in \operatorname{Near}(i) \cup \operatorname{Random}(i)} \delta_{i j}^{2}}}
$$

na qual, $N e a r(i)$ corresponde ao subconjunto de instância mais próximas da instância $i$ em cada iteração e $\operatorname{Random}(i)$ corresponde a um subconjunto aleatório de instâncias de mesmo tamanho que Near $(i)$.

Nos níveis mais baixos, o sistema converge rápido devido ao número reduzido de instâncias. Nos níveis mais altos, o sistema converge após poucas iterações devido ao fato de que as instâncias que vieram do nível de baixo já estão próximos de suas posições finais, o que acelera bastante o processo de projeção e possibilita sua implementação de forma paralela.

Por fim, uma última técnica global bastante rápida e simplista é a Random Projection [1]. Na Random Projection, o conjunto $\mathcal{X}$ é mapeado utilizando uma matriz aleatória $R_{p \times m}$ cujas colunas possuem tamanho unitário. Na forma matricial, essa transformação é dada por $Y_{n \times q}=X_{n \times p} R_{p \times q}$. O ponto central da técnica é a escolha da matriz $R$. Geralmente, os elementos $r_{i j}$ de $R$ são distribuídos segundo uma Gaussiana. Achlioptas [1] mostrou que essa distribuição pode ser substituída por algo mais simples, dado por

$$
r_{i j}=\cdot\left\{\begin{aligned}
\sqrt{3}, & \text { com probabilidade } \frac{1}{6} \\
0, & \text { com probabilidade } \frac{2}{3} \\
-\sqrt{3}, & \text { com probabilidade } \frac{1}{6} .
\end{aligned}\right.
$$

A Random Projection é uma das técnicas globais mais rápidas que existem, mas sua precisão, em termos da preservação das relações de similaridade e vizinhança, é bastante afetada.

Em geral, técnicas globais são precisas na preservação da média dos relacionamentos de distâncias, mas falham na preservação dos relacionamentos de vizinhanças principalmente quando são considerados espaços de alta dimensão esparsos. Nesses espaços, normalmente as instâncias pertencem a manifolds locais e são relacionadas a poucas instâncias que pertencem ao manifold [39]. Ou seja, a maioria das instância são muito dissimilares entre si com apenas algumas poucas compartilhando propriedades comuns [39]. Nesses casos é preferível utilizar técnicas locais, apresentadas a seguir. 


\subsection{Técnicas Locais}

Uma das primeiras tentativas de se criar uma técnica local foi apresentada por Chalmers [10]. Diferente das abordagens apresentadas anteriormente, para cada instância a abordagem de Chalmers busca preservar no espaço transformado as distâncias para duas diferentes listas de instâncias, uma contendo os vizinhos mais próximos no espaço original e outra com instâncias escolhidas de forma aleatória.

A primeira lista $V_{i}$ armazena as referências às instâncias vizinhas de $\mathbf{x}_{i}$, tendo tamanho máximo $V_{\max }$. Junto com $V_{i}$ é mantido um valor, $\max D i s t_{i}$, que é a maior distância multidimensional entre $\mathbf{x}_{i}$ e qualquer membro de $V_{i}$. Enquanto a lista de vizinhos é mantida entre iterações, a segunda lista é construída a cada passo. Essa lista é formada por um subconjunto $S_{i}$ de instâncias escolhidas aleatoriamente que não pertencem a $V_{i}$. O tamanho da mesma é definido por uma constante $S_{\max }$.

Em cada iteração, instâncias são selecionadas para serem inseridas em $S_{i}$. Toda vez que uma instância é selecionada, a dissimilaridade $\delta\left(\mathbf{x}_{i}, \mathbf{x}_{j}\right)$ é calculada. Se $\delta\left(\mathbf{x}_{i}, \mathbf{x}_{j}\right)<$ $\max _{i s t}{ }_{i}$, então $\mathbf{x}_{j}$ é inserida na lista $V_{i}$ ao invés de $S_{i}$. Esse procedimento é repetido até que a lista $S_{i}$ tenha $S_{\max }$ instâncias. Quando isso ocorrer, o posicionamento de $\mathbf{x}_{i}$ é calculado considerando um número limitado de instâncias (menor ou igual a $V_{\max }+S_{\max }$ ).

Conforme a amostragem aleatória continua, a lista $S_{i}$ evolui na direção de conter as $S_{\max }$ instâncias mais próximas de $\mathbf{x}_{i}$. Uma vez que adições de instâncias às listas $S_{i}$ tenham terminado, o processo de criação do layout geralmente se encerra entre $n$ a $3 n$ iterações, onde uma iteração envolve o posicionamento das $n$ instâncias do conjunto de dados.

Embora consiga uma melhor preservação das distâncias em pequenas vizinhanças, a abordagem apresentada por Chalmers é cara computacionalmente, $O\left(n^{2}\right)$. Outras técnicas baseadas na apresentada por Chalmers alcançaram complexidades menores, $O\left(\sqrt[4]{n^{5}}\right)$ [41] e $O(n \log n)$ [31], usando amostragem e interpolação. Essas abordagens melhoram a complexidade mas diminuem a precisão na preservação de estruturas locais de distância.

Empregando uma abordagem diferente, a técnica Local Linear Embedding (LLE) [59] realiza a redução de dimensionalidade baseada na suposição de que localmente os dados são lineares. Isto significa que pequenos pedaços em $\mathbb{R}^{p}$ devem ser aproximadamente iguais a pequenos pedaços dos dados finais em $\mathbb{R}^{q}$. Portanto, relações locais entre dados em $\mathbb{R}^{p}$ que são invariantes sobre rotação, translação e escala deveriam ser (aproximadamente) válidas em $\mathbb{R}^{q}$. Usando este princípio, o procedimento para encontrar as coordenadas de baixa dimensão é realizado em três passos: (1) encontrar os vizinhos mais próximos de cada instância; (2) expressar cada instância $\mathbf{x}_{i}$ como uma combinação linear das outras instâncias, isto é, $\mathbf{x}_{i}=$ $\sum_{j} w_{i j} \mathbf{x}_{j}$, onde $\sum_{j} w_{i j}=1$ e $w_{i j}=0$ se $\mathbf{x}_{j}$ não é um vizinho próximo de $\mathbf{x}_{i}$; (3) encontrar as coordenadas $q$-dimensionais de cada instância no espaço $p$-dimensional usando os pesos encontrados no passo 2. 
No passo 2, a matriz de pesos $W$, cujas entradas são $w_{i j}$, é encontrada minimizando-se uma aproximação do erro quadrático pela equação

$$
\operatorname{erro}(W)=\sum_{i}\left(\mathbf{x}_{i}-\sum_{j} w_{i j} \mathbf{x}_{j}\right)^{2}
$$

onde $W$ pode ser encontrada resolvendo-se um problema de mínimos quadrados.

A redução de dimensionalidade acontece no passo 3. Dada a matriz de pesos e o número de dimensões $q$, o algoritmo constrói um mapeamento preservando a vizinhança dos dados em um espaço de menor dimensão. Para isso, minimiza-se

$$
\operatorname{erro}(Y)=\sum_{i}\left(\mathbf{y}_{i}-\sum_{j} w_{i j} \mathbf{y}_{j}\right)^{2} .
$$

Para a minimização dessa equação, um método baseado em autovetores é empregado. Mais precisamente, resolve-se um sistema $n \times n$ dado pela Equação (7). Assim, a técnica LLE é de complexidade $O\left(n^{2}\right)$. Porém, métodos para cálculo de autovetores esparsos podem ser empregados de forma a reduzir essa complexidade.

Outra técnica local, foi apresentada por Tenenbaum et al., conhecida como Isometric Feature Mapping (ISOMAP) [66]. Nessa as relações de distância entre as instâncias multidimensionais são alteradas antes que a verdadeira projeção seja criada. A ideia da ISOMAP é, ao invés de utilizar distâncias Euclidianas ou outra dissimilaridade entre as instâncias multidimensionais, utilizar distâncias geodésicas. Dados um grafo $G(A, V)$, no qual $A$ denota suas arestas e $V$ seus vértices, a distância geodésica entre dois vértices $i, j \in V$ é dada pelo menor caminho entre $i$ e $j$ em $G$. Assim, para se calcular tais distâncias é criado um grafo onde $V$ são as instâncias multidimensionais e as arestas $A$ ligam cada instância aos seus $k$ vizinhos mais próximos, ponderadas de acordo com as dissimilaridades. Fazendo uso desse grafo, as novas dissimilaridades são calculadas pelo caminho mais curto entre pares de vértices desse grafo. Na ISOMAP a projeção final é obtida usando-se a Classical Scaling considerando as distâncias geodésicas.

Uma versão mais eficiente da ISOMAP utiliza a abordagem de amostragem e interpolação para reduzir o número de distâncias calculadas. Essa abordagem é conhecida como Landmarks ISOMAP (LISOMAP) [62], e possibilita que grandes conjuntos de dados sejam processados.

Um exemplo de técnica que apresenta boa precisão em termos de preservação de relações locais de vizinhança é a Least Square Projection (LSP) [48]. A LSP consiste de 
três passos principais. Primeiro, um grafo de vizinhanças entre as instâncias de $\mathcal{X}$ é definido. Em seguida, seleciona-se um subconjunto $\mathcal{X}^{\prime} \subset \mathcal{X}$ (com $\left|\mathcal{X}^{\prime}\right|=c$ ), os pontos de controle, e gera-se a projeção destes com uma técnica convencional, como o Classical Scaling. Por fim, as instâncias restantes são projetadas interpolando as já projetadas usando um sistema de equações lineares. Este sistema é montado de forma a estabelecer restrições de posicionamento que respeitem as relações de vizinhanças descritas pelo grafo encontrado no primeiro passo. Seja $V_{i}=\left\{\mathbf{x}_{i_{1}}, \ldots, \mathbf{x}_{i_{k_{i}}}\right\}$ o conjunto de $k_{i}$ pontos vizinhos de $\mathbf{x}_{i}$. Na LSP o posicionamento de cada $\mathbf{y}_{i}$ é dado pela seguinte equação

$$
\mathbf{y}_{i}-\sum_{\mathbf{x}_{j} \in V_{i}} \alpha_{i j} \mathbf{y}_{j}=0, \quad 0 \leq \alpha_{i j} \leq 1, \sum_{\mathbf{x}_{j} \in V_{i}} \alpha_{i j}=1 .
$$

Se resolvermos a Equação (8) para todas as instâncias de $\mathcal{X}$, cada $\mathbf{x}_{i}$ será mapeado no fecho convexo das imagens das instâncias em $V_{i}$. Particularmente, quando $\alpha_{i j}=\frac{1}{k_{i}}$ temos $\mathbf{y}_{i}$ no centroide das imagens de $V_{i}[68,21]$. A Equação (8) resulta em um conjunto de sistemas lineares que torna possível o cálculo das coordenadas $\tilde{p}_{i}: L \mathrm{x}_{1}=0, L \mathrm{x}_{2}=0, \ldots, L \mathrm{x}_{d}=0$, onde $\mathrm{x}_{1}, \mathrm{x}_{2}, \ldots, \mathrm{x}_{d}$ são vetores contendo as coordenadas cartesianas dos pontos e $L$ é uma matriz Laplaciana $n \times n$, cujas entradas são dadas por

$$
l_{i j}=\left\{\begin{aligned}
1, & \text { se } i=j \\
-\alpha_{i j}, & \text { se } p_{j} \in V_{i} \\
0, & \text { caso contrário. }
\end{aligned}\right.
$$

Os pontos de controle são, então, utilizados para adicionar informação geométrica ao sistema. Eles são inseridos no sistema linear como novas equações na matriz. As coordenadas cartesianas dos pontos de controle são adicionadas do lado direito do sistema, levando a um vetor não-nulo. Assim é possível re-escrever a Equação (8) da seguinte forma: $A \mathrm{x}=b$, onde $A$ é uma matriz $(n+n c) \times n$ dada por

$$
A=\left(\begin{array}{c}
L \\
C
\end{array}\right), \quad c_{i j}= \begin{cases}1, & \mathbf{x}_{j} \in \mathcal{X}^{\prime} \\
0, & \text { caso contrário }\end{cases}
$$

e $b$ é o vetor

$$
b_{i}=\left\{\begin{array}{rl}
0 & i \leq n \\
x_{c_{i} k} & n<i \leq n+c
\end{array}\right.
$$

onde $x_{c_{i} k}$ é uma das coordenadas cartesianas do ponto de controle $\mathbf{x}_{c_{i}}$.

Embora a LSP apresente bons resultados em termos de preservação de vizinhança, o operador Laplaciano necessita da solução de sistema linear com $n$ variáveis, o que a torna computacionalmente impraticável para grandes conjuntos de dados. 
Como forma de contornar esse problema, Paulovich et al. [45] apresentam a técnica Piecewise Laplacian Projection (PLP). A PLP resolve pequenos sistemas lineares ao invés de apenas um muito grande. Isso é feito particionando o conjunto de dados em pequenos grupos e aplicando a LSP para como um desses conjuntos de forma independente. A coerência geométrica entre as projeções desses grupos é alcançada projetando os pontos de controle desses grupos em conjunto.

Com essa subdivisão em sistemas lineares menores, a PLP apresenta custo computacional menor quando comparada com a LSP. No entanto, perde em termos de preservação de vizinhança.

Outra técnica que faz uso de instâncias representativas em um passo anterior para a geração da projeção final é a Local Affine Multidimensional Projection (LAMP) [30]. O diferencial dessa técnica é a possibilidade de manipulação dos pontos de controle no espaço visual pelo usuário ao mesmo tempo que possui custo computacional baixo e boa precisão em termos de preservação de dissimilaridades nos mapeamentos. Partindo de uma projeção gerada para uma amostra dos conjuntos de dados, o usuário pode manipular os elementos no espaço visual, mudando as relações de similaridade. A projeção dos demais elementos é gerada a partir destes elementos.

A solução adotada pela LAMP para o mapeamento de cada instância é encontrar uma função linear para cada uma delas. O problema é definido em termos das coordenadas de posicionamento das instâncias representativas, e o mapeamento de cada $\mathbf{x}_{i}$ é a transformação $f_{\mathbf{x}_{i}}(\mathbf{x})=\mathbf{x} M+\mathbf{t}$ que otimiza

$$
\begin{aligned}
\min _{f_{\mathbf{x}_{i}}} & \sum_{j=1}^{c} \alpha_{j}\left\|f_{\mathbf{x}_{i}}\left(\mathbf{x}_{j}^{\prime}\right)-\mathbf{y}_{j}^{\prime}\right\|^{2} \\
\text { sujeito a } & M^{T} M=I,
\end{aligned}
$$

onde $\alpha_{j}=\frac{1}{\left\|\mathbf{x}_{j}^{\prime}-\mathbf{x}_{i}\right\|^{2}}$ são pesos adicionados para que instâncias representativas mais similares influenciem mais no mapeamento de $\mathbf{x}_{i}$, enquanto que as menos similares irão influenciar menos. Além disso, remover a influência de instâncias representativas mais distantes é o que permite que o mapeamento seja realmente local.

A restrição de ortogonalidade adicionada ao problema da Equação (9) impõe uma transformação que só rotaciona e translada os dados. Uma característica importante é que efeitos de escala ou cisalhamento não são inseridos, ajudando a minimizar as diferenças entre as dissimilaridades originais e as distâncias na representação visual. Além disso, a distorção adicionada pelo posicionamento de instâncias representativas é suavizada. Uma solução pode ser encontrada utilizando um método de mínimos quadrados tradicional, como visto em outras técnicas, mas essa abordagem pode propagar as distorções [30]. 
A solução para a minimização da Equação (9) é reescrevê-la em forma de matriz, dando origem a um problema de Procrustes Ortogonal [22], o qual possui solução conhecida. Basta tornar a derivada parcial de $\mathbf{t}$ igual a zero e reescrever $\mathbf{t}$ em termos de $M \operatorname{com} \mathbf{t}=$ $\overline{\mathbf{y}}^{\prime}-\overline{\mathbf{x}}^{\prime} M$, onde

$$
\overline{\mathbf{x}}^{\prime}=\frac{\sum_{j=1}^{c} \alpha_{j} \mathbf{x}_{j}^{\prime}}{\sum_{j=1}^{c} \alpha_{j}}, \quad \overline{\mathbf{y}}^{\prime}=\frac{\sum_{j=1}^{c} \alpha_{j} \mathbf{y}_{j}^{\prime}}{\sum_{j=1}^{c} \alpha_{j}} .
$$

De volta a Equação (9), temos

$$
\min _{f_{\mathbf{x}_{i}}} \sum_{j=1}^{c} \alpha_{j}\left\|f_{\mathbf{x}_{i}}\left(\hat{\mathbf{x}}_{j}\right)-\hat{\mathbf{y}}_{j}\right\|^{2}
$$

sujeito a $M^{T} M=I$,

onde $\hat{\mathbf{x}}_{j}=\mathbf{x}_{j}^{\prime}-\overline{\mathbf{x}}^{\prime}$ e $\hat{\mathbf{y}}_{j}=\mathbf{y}_{j}^{\prime}-\overline{\mathbf{y}}^{\prime}$. Por fim, escrevendo a Equação (10) na forma matricial, temos

$$
\begin{aligned}
\text { minimização } & \|A M-B\|_{F} \\
\text { sujeito a } & M^{T} M=I,
\end{aligned}
$$

onde $\|\cdot\|_{F}$ representa a norma de Frobenius e as matrizes $A$ e $B$ são dadas por:

$$
A=\left[\begin{array}{c}
\sqrt{\alpha_{1}} \hat{\mathbf{x}}_{1}^{T} \\
\sqrt{\alpha_{2}} \hat{\mathbf{x}}_{2}^{T} \\
\vdots \\
\sqrt{\alpha_{c}} \hat{\mathbf{x}}_{c}^{T}
\end{array}\right], \quad B=\left[\begin{array}{c}
\sqrt{\alpha_{1}} \hat{\mathbf{y}}_{1}^{T} \\
\sqrt{\alpha_{2}} \hat{\mathbf{y}}_{2}^{T} \\
\vdots \\
\sqrt{\alpha_{c}} \hat{\mathbf{y}}_{c}^{T}
\end{array}\right] .
$$

Como mencionado anteriormente, a Equação (11) possui solução conhecida, fazendo $A^{T} B=$ $U D V^{T}$, onde $U D V^{T}$ corresponde à decomposição em valores singulares (do inglês, SVD) de $A^{T} B$, temos que $M=U V^{T}$. Agora é possível calcular $f_{\mathbf{x}_{i}}$, a qual retorna $\mathbf{y}_{i}$,

$$
f_{\mathbf{x}_{i}}(\mathbf{x})=\left(\mathbf{x}-\overline{\mathbf{x}}^{\prime}\right) M+\overline{\mathbf{y}}^{\prime} .
$$

Os mapeamentos locais para cada instância tornam a LAMP uma técnica eficiente e eficaz. Respostas mais rápidas e a possibilidade de modificações locais de mapeamento permitem que a técnica seja utilizada em várias aplicações [30].

Para tratar espaços de alta dimensão esparsos, como aqueles que representam os conjuntos de coleções de documentos, é apresentada a técnica chamada Local Convex Hull (LoCH) [20]. Nesses espaços as instâncias de dados são organizadas em manifolds locais, com a maioria das instâncias sendo dissimilares entre si [39]. 
A técnica LoCH pode ser dividida em três passos principais: primeiro, para cada instância encontra-se os seus $k$ vizinhos mais próximos; em seguida, instâncias representativas são escolhidas como amostras e projetadas no plano; por fim, as instâncias restantes são interpoladas considerando as amostras e um processo iterativo é executado para posicionar as instâncias o mais próximo possível de seus vizinhos mais próximos.

Para encontrar os vizinhos mais próximos, os autores aplicam uma estratégia simples baseada em agrupamento. Para a amostra de instâncias representativas, a técnica aproveita os medóides do passo anterior. De posse das amostras, as mesmas são posicionadas no plano utilizando uma técnica global que preserva o máximo possível dos relacionamentos de distâncias definidos no espaço original.

O último passo é a principal contribuição apresentada pela $\mathrm{LoCH}$, a aproximação do fecho convexo. Seja $N_{i}=\left\{\mathbf{x}_{i_{1}}, \mathbf{x}_{i_{2}}, \ldots, \mathbf{x}_{i_{k_{i}}}\right\}$ a lista contendo os $k_{i}$ vizinhos mais próximos de $\mathbf{x}_{i}$. Qualquer posição dentro do fecho convexo no espaço $p$-dimensional composto pelas instâncias de $N_{i}$ pode ser obtido por meio da equação

$$
\hat{\mathbf{y}}_{i}=\sum_{\mathbf{x}_{j} \in N_{i}} \alpha_{j} \mathbf{y}_{j}
$$

$\operatorname{com} \alpha_{j} \geq 0$ e $\sum \alpha_{j}=1$

Considerando todas as possíveis posições dentro do fecho convexo, a técnica busca encontrar aquela que preserva no espaço transformado os relacionamentos de distância entre $\mathbf{x}_{i}$ e seus vizinhos mais próximos $N_{i}$. Assim, $\alpha_{i}$ é definido como sendo inversamente proporcional às distâncias no espaço original

$$
\alpha_{j}=\left(\delta\left(\mathbf{x}_{i}, \mathbf{x}_{j}\right) \cdot \sum_{\mathbf{x}_{k} \in N_{i}} 1 / \delta\left(\mathbf{x}_{i}, \mathbf{x}_{k}\right)\right)^{-1}
$$

sempre que $\delta\left(\mathbf{x}_{i}, \mathbf{x}_{k}\right)=0, \hat{\mathbf{y}}_{i}=\mathbf{y}_{k}$.

A técnica move cada ponto $\mathbf{y}_{i}$ em direção a $\hat{\mathbf{y}}_{i}$. Como não é possível garantir que todas as instâncias residem no fecho convexo de seus vizinhos mais próximos, não é possível afirmar que $\mathbf{y}_{i}$ é igual a $\hat{\mathbf{y}}_{i}$. Em vez disso, $\mathbf{y}_{i}$ é movido em direção à posição $\widetilde{\mathbf{y}}_{i}$ que reside na direção do vetor de $\hat{\mathbf{y}}_{i}$ até a posição atual de $\mathbf{x}_{i}$. Seja $\overrightarrow{\mathbf{v}}$ esse vetor, $\widetilde{\mathbf{y}}_{i}$ é calculado por

$$
\widetilde{\mathbf{y}}_{i}=\hat{\mathbf{y}}_{i}+\gamma_{i} \frac{\overrightarrow{\mathbf{v}}}{\|\overrightarrow{\mathbf{v}}\|}
$$

onde $\gamma_{i}$ é definida para preservar o máximo possível a distância original entre $\mathbf{x}_{i}$ e as instâncias em $N_{i}$. Seja $\overrightarrow{\mathbf{u}}_{j}$ o vetor de $\hat{\mathbf{y}}_{i}$ para um ponto $\mathbf{y}_{j}$ que representa a instância $\mathbf{x}_{j} \in N_{i}$ e $\tau_{j}$ 
a magnitude da projeção de $\overrightarrow{\mathbf{u}}_{j}$ em $\overrightarrow{\mathbf{v}}$, $\operatorname{com} \tau_{j}=(\overrightarrow{\mathbf{v}} /\|\overrightarrow{\mathbf{v}}\|) \cdot \overrightarrow{\mathbf{u}}_{j}$. O valor de $\gamma_{i}$ que melhor preserva a distância $\delta\left(\mathbf{x}_{i}, \mathbf{x}_{j}\right)$, considerando todas as instâncias em $N_{i}, \gamma_{i}$ é calculada por

$$
\gamma_{i}=\frac{1}{k_{i}} \sum_{\mathbf{x}_{j} \in N_{i}}\left(\tau_{j}+\sqrt{\delta\left(\mathbf{x}_{i}, \mathbf{x}_{j}\right)^{2}+\tau_{j}^{2}-\| \overrightarrow{\mathbf{u}}_{j}} \|^{2}\right) .
$$

Utilizando essas equações a técnica LoCH move iterativamente cada ponto $\mathbf{y}_{i}$ em direção a $\widetilde{\mathbf{y}}_{i}$ até que nenhum movimento seja possível ou até que um número máximo de iterações seja atingido. Então, a posição $\mathbf{y}_{i}^{t}$ de $\mathbf{x}_{i}$ na $t$-ésima iteração é atualizada por

$$
\mathbf{y}_{i}^{t}=\left[1-\omega_{i}(t)\right] \mathbf{y}_{i}^{t-1}+\omega_{i}(t) \widetilde{\mathbf{y}}_{i}
$$

com $\omega_{i}(t)$ variando em $[0,1]$, definindo a liberdade de movimento de $\mathbf{y}_{i}$ na iteração $t$. Valores maiores de $\omega_{i}(t)$ fazem $\mathbf{y}_{i}$ mover mais.

Os resultados mostram que a técnica LoCH é capaz de separar grupos de instâncias similares embutidos em espaços esparsos de alta dimensionalidade, definindo bem as bordas entre eles.

Diferentemente das técnicas anteriores, a NeRV [70] utiliza modelos probabilísticos para encontrar o mapeamento no espaço de baixa dimensão. Para isso, os conceitos de precisão e revocação são trazidos para o contexto de projeções multidimensionais. Assim como no caso de recuperação de informações, estamos interessados em avaliar o quão bom é um mapeamento em obter resultados relevantes para uma certa consulta utilizando a precisão e a revocação obtidas. A técnica NeRV tem por objetivo maximizar um equilíbrio entre as duas medidas. Este equilíbrio é definido pelo usuário, que indica o custo relativo entre essas.

Considerando que um usuário inspeciona um mapeamento e procura por vizinhos das instâncias, uma consulta pode ser vista como a escolha de uma instância $i$ e a observação de instâncias vizinhas a esta. Dessa forma, a probabilidade de uma instância $j$ ser vizinha de $i$ no mapeamento é definida como

$$
q_{j \mid i}=\frac{\exp \left(-\frac{\left\|\mathbf{y}_{i}-\mathbf{y}_{j}\right\|^{2}}{\sigma_{i}^{2}}\right)}{\sum_{k \neq i} \exp \left(-\frac{\left\|\mathbf{y}_{i}-\mathbf{y}_{k}\right\|^{2}}{\sigma_{i}^{2}}\right)},
$$

onde os valores $\sigma_{i}$ controlam indiretamente o tamanho das vizinhanças em $\mathcal{X}$. De forma análoga, define-se a probabilidade de uma instância $j$ ser vizinha de uma instância $i$ no espaço multidimensional como

$$
p_{j \mid i}=\frac{\exp \left(-\frac{\delta\left(\mathbf{x}_{i}, \mathbf{x}_{j}\right)^{2}}{\sigma_{i}^{2}}\right)}{\sum_{k \neq i} \exp \left(-\frac{\delta\left(\mathbf{x}_{i}, \mathbf{x}_{k}\right)^{2}}{\sigma_{i}^{2}}\right)} .
$$


Em [70], sugere-se ajustar o valor de cada $\sigma_{i}$ de forma que a distribuição de probabilidades de $i$ tenha entropia igual a $\log k, \operatorname{com} k$ definido previamente. Isso faz com que $k$ se torne, de certa forma, um limitante superior para o número de instâncias nas vizinhanças.

Com as distribuições de probabilidades definidas, precisão e revocação podem ser encontradas em termos destas. Por meio da divergência de Kullback-Leibler [37], dada por

$$
D\left(p_{\cdot \mid i}, q_{\cdot \mid i}\right)=\sum_{j \neq i} p_{j \mid i} \log \frac{p_{j \mid i}}{q_{j \mid i}},
$$

pode-se demonstrar que, para uma consulta $i, D\left(p_{\cdot \mid i}, q_{\cdot \mid i}\right)$ equivale à revocação, enquanto que $D\left(q_{\cdot \mid i}, p_{\cdot \mid i}\right)$ equivale à precisão [70]. Como não é possível maximizar ambas as medidas simultaneamente, um custo relativo $\lambda$ é utilizado para determinar qual das duas medidas se torna mais importante na maximização. Assim, a técnica NeRV consiste em maximizar a função de custo

$$
C_{\mathrm{NeRV}}=\lambda \sum_{i} \sum_{j \neq i} p_{j \mid i} \log \frac{p_{j \mid i}}{q_{j \mid i}}+(1-\lambda) q_{j \mid i} \log \frac{q_{j \mid i}}{p_{j \mid i}} .
$$

Um resultado notável vindo desta formulação surge quando consideramos o caso em que $\lambda=1$. Assumindo tal valor na Equação (22), obtemos a função de custo da técnica SNE [24]. Consequentemente, ganhe-se a interpretação de que a SNE maximiza a revocação em seus mapeamentos.

Ainda em [70], discute-se a possibilidade de alterar as probabilidades $p_{j \mid i}$ e $q_{j \mid i}$ para probabilidades conjuntas $p_{i j}$ e $q_{i j}$, respectivamente. As novas probabilidades são dadas por

$$
p_{i j}=\frac{1}{2 n}\left(p_{i \mid j}+p_{j \mid i}\right),
$$

e

$$
q_{i j}=\frac{\left(1+\left\|\mathbf{y}_{i}-\mathbf{y}_{j}\right\|^{2}\right)^{-1}}{\sum_{k \neq l}\left(1+\left\|\mathbf{y}_{k}-\mathbf{y}_{l}\right\|\right)^{-1}},
$$

com $\sum_{i, j} p_{i j}=1$ e $\sum_{i, j} q_{i j}=1$. As novas probabilidades, baseadas nas modificações da SNE propostas na técnica t-SNE [69], lidam com dois tipos de problemas presentes na formulação anterior. A primeira, força que mesmo instâncias de $\mathcal{X}$ que são espúrias possuam outras instâncias do conjunto como vizinhas. A segunda, uma distribuição-t de grau de liberdade 1, tem a intenção de aliviar o problema de aglomeramento, isto é, quando os grupos de instâncias presentes nos dados são posicionados, os pontos moderadamente distantes não afetam o posicionamento dos outros pontos no mapeamento final. 
A função de custo associada às novas distribuições é otimizada pela variante da NeRV, a t-NeRV, e é dada por

$$
C_{\mathrm{t}-\mathrm{NeRV}}=\lambda \sum_{i} \sum_{j \neq i} p_{i j} \log \frac{p_{i j}}{q_{i j}}+(1-\lambda) q_{i j} \log \frac{q_{i j}}{p_{i j}} .
$$

Analogamente à Equação (22), a Equação (25) se torna a função de custo da técnica tSNE [69] quando temos $\lambda=1$, novamente levando à interpretação de que a t-SNE maximiza a revocação de pontos de consulta e seus vizinhos, dada a natureza conjunta das distribuições de probabilidade.

As técnicas locais buscam preservar as distâncias em pequenas vizinhanças, alcançando melhores resultados quando se trata de preservação das relações de vizinhança estabelecidas no espaço original. Se o objetivo da análise for a separação de grupos e a definição de suas fronteiras, as técnicas locais representam a melhor escolha, principalmente para conjuntos de dados de alta dimensionalidade [20]. No entanto, a maioria das técnicas locais possui como característica a tendência de formar aglomerados compactos, quando a distribuição das distâncias define grupos bem distintos nos dados. Isso pode dificultar a exploração visual, devido à grande sobreposição das instâncias no espaço visual.

\section{Aplicações}

Nessa seção diferentes aplicações de projeções multidimensionais são apresentadas, começando com análise de coleções de documentos, passando pela exploração de coleções de músicas, pela análise de dados de sensores e biosensores e terminando com a exploração de espaços de características.

\subsection{Visualização de Coleção de Documentos}

Uma das aplicações que tem despertado interesse da comunidade científica é a exploração visual de coleções de documentos. O emprego de projeções multidimensionais pode ser bastante efetivo nesse processo, resultando no que é conhecido como "mapa de documentos" [48]. Mapas podem suportar uma variedade de tarefas exploratórias, conectando usuários com seus respectivos mapas cognitivos do espaço de informação visual [12] enquanto evitam algumas das complexidades inerentes ao espaço de informação subjacente. Assim, eles facilitam o acesso a coleções complexas de documentos e melhoram a efetividade de resolver problemas reais de tarefas de gerenciamento de conhecimento [7].

Entre as abordagens mais conhecidas para a criação de mapas de documentos baseadas em projeções multidimensionais podemos destacar: 
- os Mapas Cartográficos de Skupin [64], cuja maior qualidade está no fato de usar uma metáfora familiar à maioria dos usuários: os mapas geográficos;

- a técnica proposta por Wise [71], conhecida como Galaxies, que emprega uma "abordagem ecológica" para a visualização de texto, a qual utiliza visualizações análogas ao céu noturno e modelos de terreno cuja interpretação é facilitada pela capacidade inerente em nosso cérebro como um resultado de nossa herança biológica.

Elas são incorporadas aos sistemas IN-SPIRE ${ }^{\mathrm{TM}}$ [55] e Infosky [3], respectivamente.

Um exemplo de visualização de coleção de documentos é apresentado na Figura 1. O mapa de documentos foi gerado utilizando a técnica LSP para uma base formada por 2.684 notícias coletadas por meio de feeds RSS da Associated Press, CNN, BBC e Reuters durante dois dias de abril de 2006. Os grupos formados nas extremidades são compostos por notícias com o mesmo assunto, como, por exemplo, gripe aviária e leis de imigração, entre outros assuntos que foram bastante comentados durante o período de coleta. As notícias que ficaram posicionadas no centro do mapa correspondem a notícias que receberam menos destaque da mídia durante o mesmo período.

A exploração de similaridades em vizinhanças permite aos usuários realizar tarefas interessantes como a busca por tópicos de interesse, busca por documentos similares, observação da estrutura principal da coleção de documentos e observação da freqüência de certos tópicos. Nesse sentido, as técnicas de projeção multidimensional se mostram bastante úteis.

\subsection{Visualização de Coleções de Músicas}

Outra aplicação interessante de técnicas de projeção multidimensional é a criação de mapas visuais que permitem a análise exploratória de coleções de músicas [45]. Entre algumas das possibilidades introduzidas por meio da análise utilizando mapas visuais, uma que pode ser considerada bastante útil é a criação de playlists, ou listas de músicas.

Nessa aplicação, o usuário começa selecionando algumas músicas de uma lista que contém o nome dos artistas e o título da música. O sistema utiliza as músicas selecionadas como amostras juntamente com outras que são selecionadas automaticamente e são muito diferentes das selecionadas pelo usuário. De posse das amostras, o sistema projeta algumas instâncias (Figura 2(a)) e permite que o usuário as manipule para formar as separações desejadas (Figura 2(b)). Por fim, o conjunto inteiro de músicas é projetado e as playlists podem ser criadas (Figura 2(c)). Esse processo é ilustrado pela Figura 2.

A utilização de técnicas de projeção multidimensional para a análise interativa de conjuntos de dados multidimensionais torna possível aplicações que dependem de intervenções 


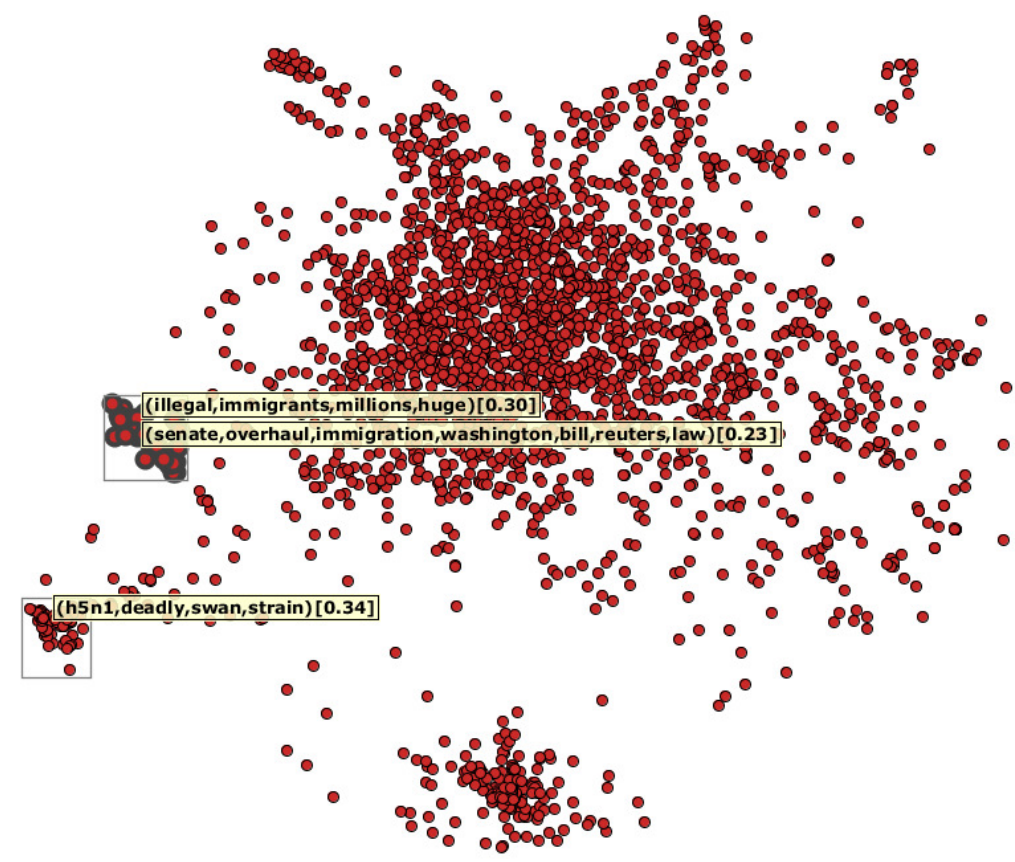

Figura 1. Mapa de documentos para uma base de notícias coletadas da Internet durante dois dias de abril de 2006.

do usuário, como, por exemplo, na organização de arquivos pessoais. Essas técnicas permitem flexibilidade, ao mesmo tempo que garantem efetividade da tarefa de análise exploratória.

\subsection{Visualização de Dados Provenientes de Sensores e Biossensores}

Um sensor ou biossensor é um dispositivo analítico que tem por objetivo distinguir diferentes substâncias e suas concentrações [40]. De forma mais específica, um determinado bio-elemento reconhece um analito ${ }^{3}$ específico e o elemento sensorial traduz suas características em sinais elétricos [57].

Um exemplo de biossensor existente são as línguas eletrônicas [57]. Esse tipo de sensor tem sido empregado para análise de diferentes substâncias que envolvem, por exemplo, a distinção de diferentes vinhos tintos [44], sucos de fruta, amostras de cerveja e chá [35], café, leite e derivados, além de detecção de traços de impurezas ou poluentes em água [57].

$\overline{{ }^{3} \text { Analito é o componente que é alvo da análise. }}$ 


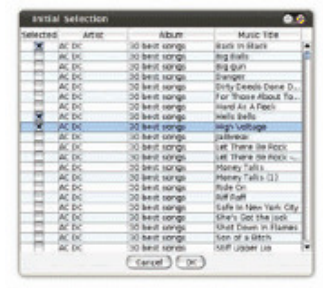

(a)
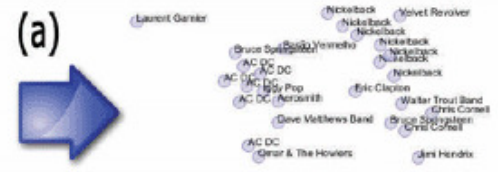

Geneses

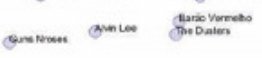

(b)
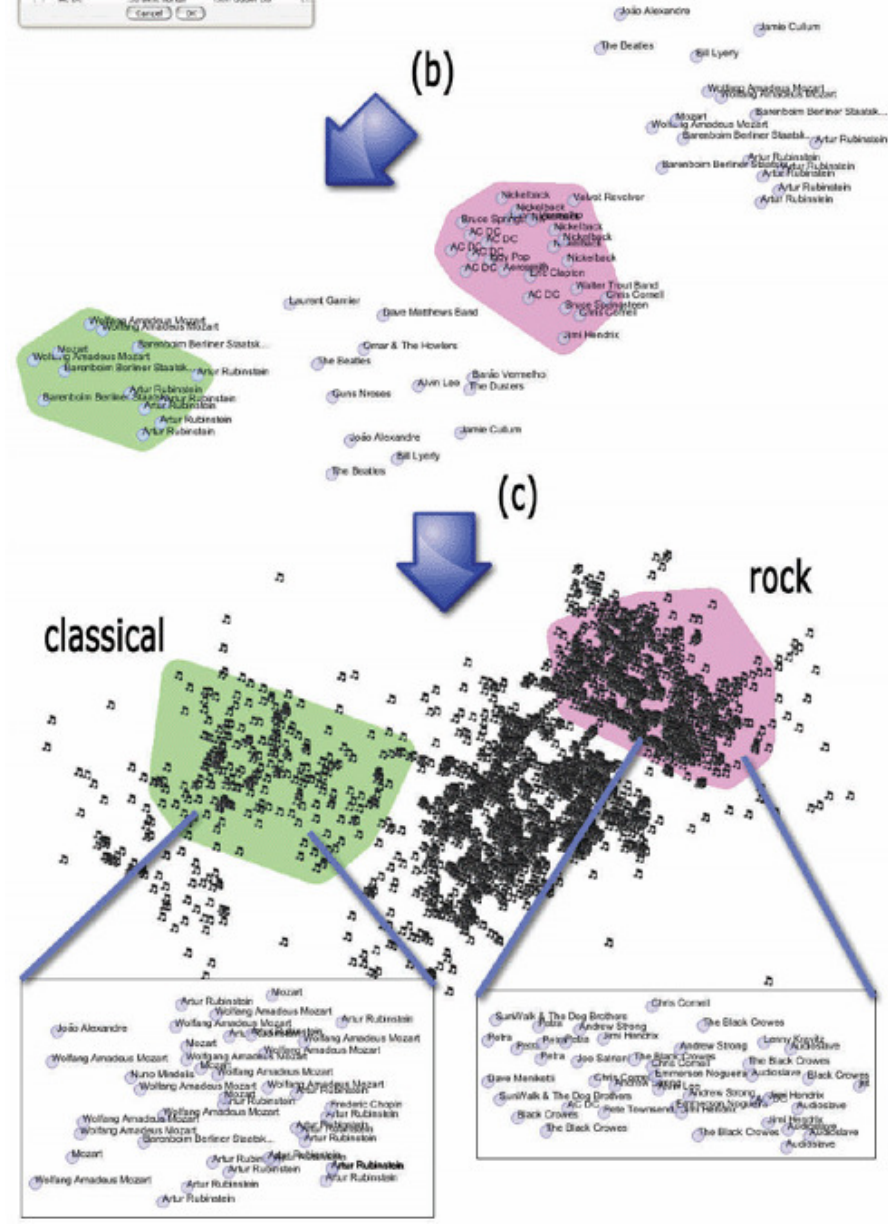

Figura 2. Processo geral do gerador de playlists. (Extraído de Paulovich et al. 2011 [45]) 
Embora sensores e biossensores sejam ferramentas bastante difundidas, a interpretação dos dados gerados não é trivial devido às características nanométricas do seu processo de fabricação. É nesse cenário que as técnicas de projeção são empregadas, ajudando os especialistas no processo de exploração dos dados. Aoki et al. [5] definem um processo que combina projeções multidimensionais com Coordenadas Paralelas [29] para comparar diferentes métodos de construção de biosensores. A Figura 3 ilustra o processo adotado. As unidades de sensoriamento, ou seja, os sensores e biossensores, coletam os dados. Em seguida, esses dados passam por algum processamento, gerando dados derivados, com, por exemplo, capacitância. Por fim, técnicas de visualização são empregadas como suporte ao entendimento desses dados.

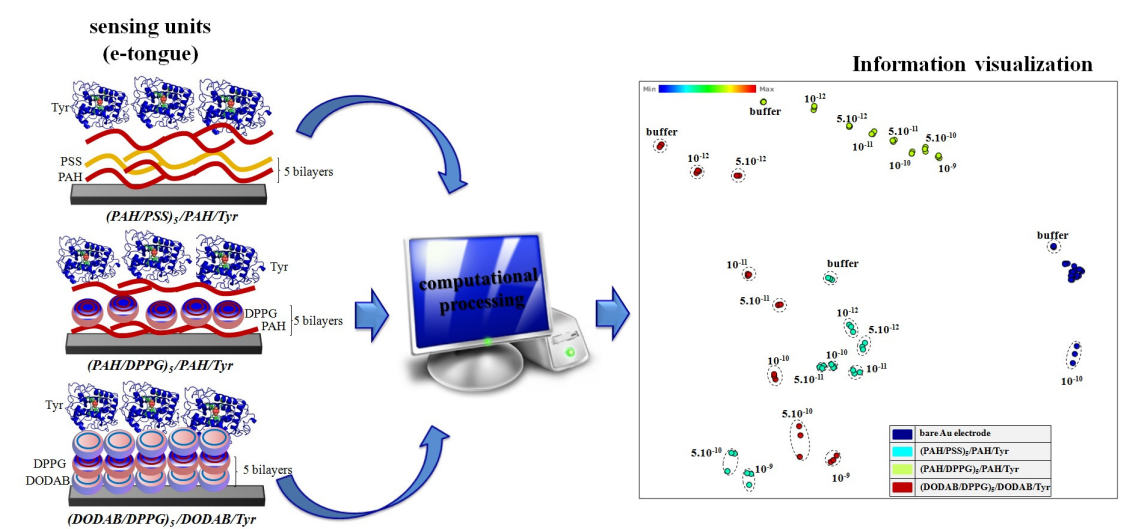

Figura 3. Ilustração do processo de análise de dados provenientes de biossensores. (Extraído de Aoki et al. 2013 [5])

A Figura 4(a) apresenta uma projeção utilizando a técnica Sammon's Mapping sobre dados obtidos a partir de diferentes unidades de sensoriamento. Círculos de mesma cor representam biossensores com processos de construção similares. É possível observar que os biossensores representados pelos círculos vermelho, ciano e verde, apresentaram uma boa separação entre as diferentes concentrações da substância em análise. Por outro lado, os sensores representados pelos círculos azuis, não apresentaram uma boa distinção. Na Figura 4(b) foi utilizada a técnica de coordenadas paralelas. Os segmentos de reta que interceptam os eixos correspondem aos elementos do conjunto de dados. É possível observar que nas baixas frequiências (eixos mais a esquerda) a separação entre diferentes concentrações é melhor identificada.

O uso de técnicas de visualização de informações na análise de dados provenientes de sensores e biossensores pode levar a resultados significantes e sem precedentes, como os 


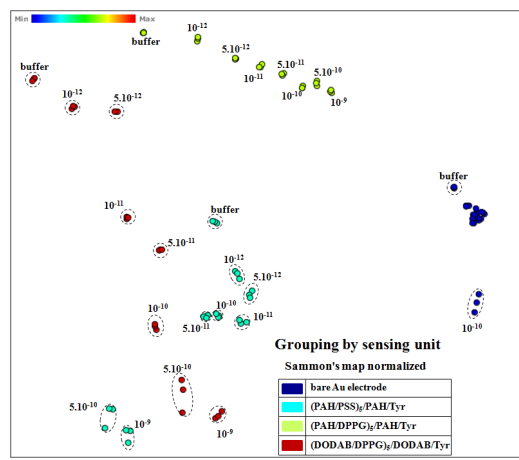

(a) Projeção multidimensional

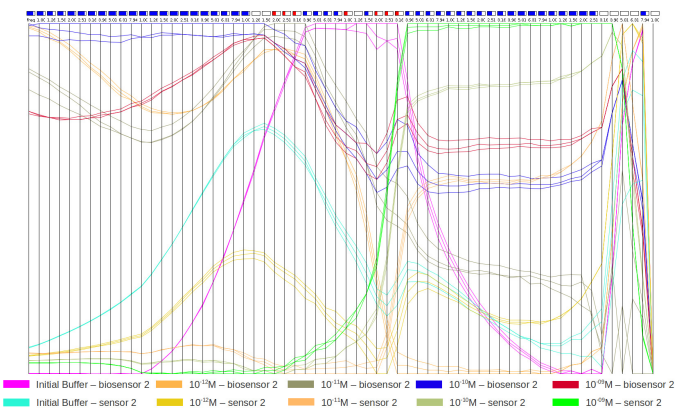

(b) Coordenadas Paralelas

Figura 4. Projeção multidimensional utilizando a técnica Sammon's Mapping sobre dados de impedância obtidos de diferentes unidades sensoriais, em (a), e Coordenadas paralelas dos dados de sensores e biossensores, em (b). (Extraído de Aoki et al. 2013 [5])

apresentados em [46].

\subsection{Visualização de Espaços de Características}

Definir um conjunto de características que representam um conjunto de dados de forma adequada não é uma tarefa fácil. A maioria das técnicas disponíveis são baseadas em medidas estatísticas ou utilizam abordagens de inteligência artificial e funcionam como "caixas pretas" para os usuários.

A utilização de representações visuais permite aos usuários ter uma visão do significado e da representatividade de características computadas a partir de diferentes algoritmos e conjuntos de parâmetros. À medida que a manipulação dos conjuntos de características e mudanças de parâmetros melhora a representação visual do espaço de característica, também promove a obtenção de uma melhor acurácia da tarefa de classificação das características computadas.

Projeções multidimensionais podem ser bastante úteis como ferramenta de suporte para a decisão de quais características devem ser utilizadas. Por exemplo, um usuário pode comparar diferentes projeções geradas a partir de diferentes conjuntos de características e escolher qual conjunto define a projeção que mais se aproxima do esperado pelo usuário, ou mesmo variar parâmetros do processo de extração de características e observar o que acontece na projeção em termos das relações de similaridade definidas entre as imagens.

De fato, a representação visual pode ser utilizada como um guia para o entendimento 
do comportamento das características no que tange o agrupamento de instâncias similares e separação das dissimilares. Contudo, cabe ressaltar que a visualização não deveria ser entendida como uma abordagem para escolher os melhores conjuntos de características para uma tarefa subjacente, como classificação, mas sim como ferramental para apoiar um melhor entendimento das possibilidades de escolha.

\section{Ferramentas}

Uma das ferramentas pioneiras para análise de dados baseada em projeções multidimensionais é conhecida como Projection Explorer (PEx) [49]. A Figura 5 mostra uma visão geral da janela principal da PEx. É possível fornecer à ferramenta diferentes tipos de entrada de dados: (1) coleções de documentos; (2) vetores multidimensionais e; (3) matrizes de distância. De forma geral, as coleções de documentos são convertidas em vetores multidimensionais, que são usados para os cálculos de distâncias, necessárias para a criação das projeções. A PEx implementa internamente diferentes técnicas de projeção, permitindo que diferentes análises sejam executadas.

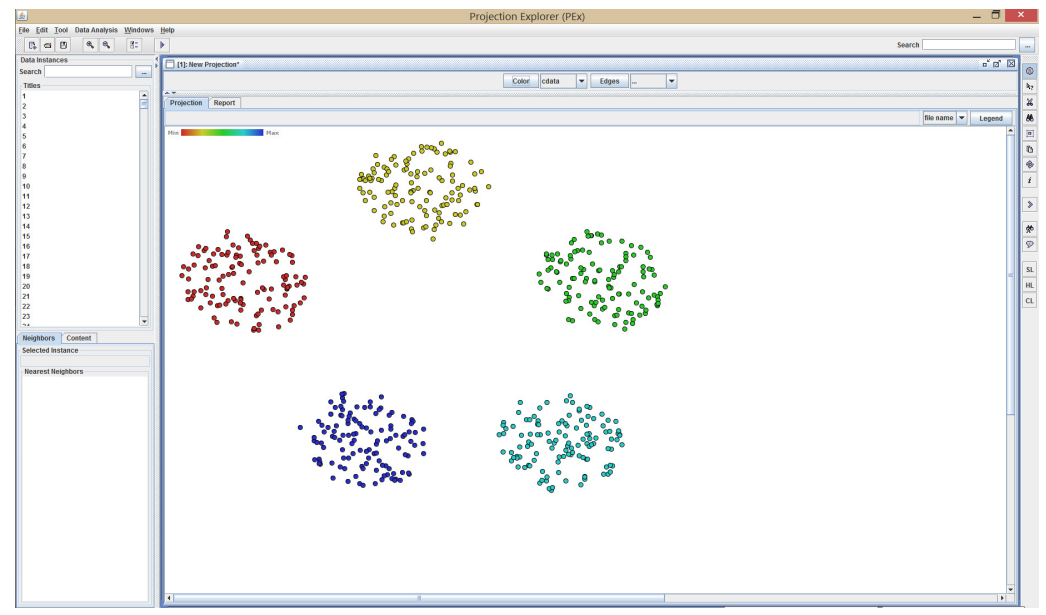

Figura 5. Janela principal da ferramenta PEx.

O usuário pode interagir com a representação gráfica criada e iniciar a exploração dos dados. Diferentes mecanismos são implementados, em especial elementos desenvolvidos para a exploração de coleções de documentos, acelerando significativamente o processo de extração de conhecimento de coleções contendo centenas ou milhares de documentos.

Para análises mais específicas, outras ferramentas foram derivadas da PEx. A PEx- 
WEB [50] busca melhorar a análise exploratória de resultados de busca na Internet. Voltada para a análise de dados provenientes de sensores e biossensores foi criada a PEx-Sensors [47]. E para a análise e exploração de coleções de imagens é possível utilizar a ferramenta PExImage [19].

Essas ferramentas estão disponíveis para download ${ }^{4}$, e são destinadas a usuários não especialistas. Para usuários mais avançados, o VisPipeline foi desenvolvido como um arcabouço de programação e é explicado a seguir.

\subsection{VisPipeline}

Escrito em Java, o VisPipeline consiste de uma coleção de componentes organizados em uma biblioteca para facilitar a comunicação entre eles. No intuito de ser flexível e ainda permitir a rápida construção de visualizações, os componentes funcionam como um pipeline, onde cada elemento é uma função bem definida. Desse modo, cada componente pode ser visto como uma unidade de processamento que aceita uma entrada e gera uma saída após sua execução.

A flexibilidade atingida por este tipo de abstração permite que seja possível substituir qualquer componente por outro que possua tipos compatíveis de entrada e saída. Por exemplo, existem componentes para leitura e escrita de conjuntos de dados em arquivos, técnicas de agrupamento de dados, projeção multidimensional e componentes para visualizar certos tipos de dados, como scatterplots.

Adicionar novas funcionalidades requer simplesmente a criação de um novo componente para uma tarefa específica, ou estender um existente que já realize parte do trabalho. Do ponto de vista da biblioteca do VisPipeline, um componente é uma classe que implementa (direta ou indiretamente) a interface Abst ract Component, definida conforme a listagem abaixo:

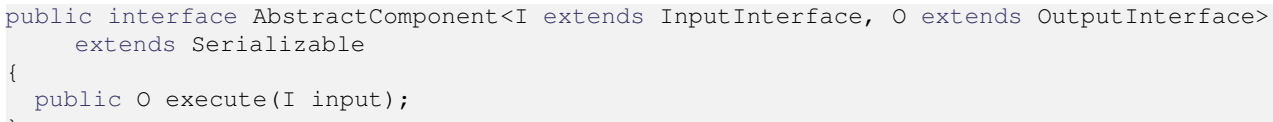

O método execute é onde o objeto recebe a entrada e deve produzir a saída. Como exemplo, a listagem abaixo mostra como seria implementado um componente para somar dois números inteiros e retornar a soma em sua saída.

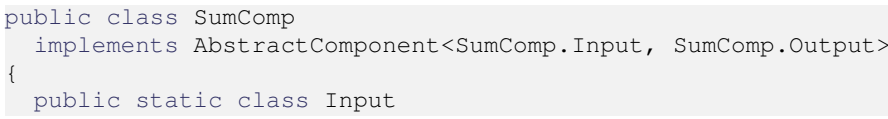

$\overline{4_{\text {http: //vis.icmc.usp.br/vicg/software }}}$ 


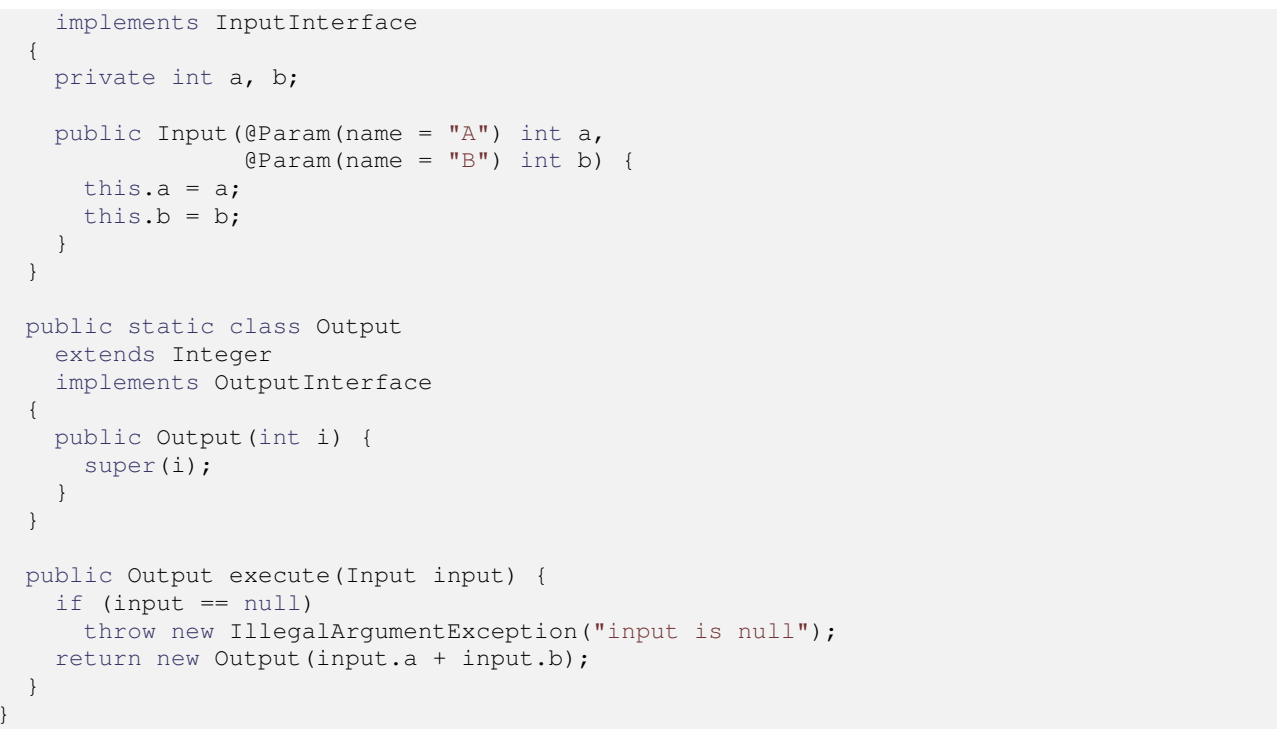

É importante notar que para definir entradas e saídas, por convenção, componentes definem classes internas chamadas Input e Output, respectivamente. Implementar tipos especializados para entradas e saídas, apesar de adicionar complexidade ao código, permite grande flexibilidade na hora de definir entradas e saídas. Ao mesmo tempo, mantém-se a ideia de que apenas um objeto é usado como entrada e apenas um objeto é usado como saída no método execute.

O VisPipeline também oferece, em uma interface gráfica especializada, uma forma de construir, interativamente, pipelines de componentes fazendo as ligações entre esses interativamente. Na Figura 6, podemos ver um pipeline para gerar uma visualização a partir de uma matriz de distância utilizando a técnica de projeção MDS e apresentando o resultado em um Frame apropriado para este tipo de mapa visual. No lado esquerdo da interface gráfica da ferramenta, encontram-se os componentes carregados e prontos para serem utilizados, apresentados em uma árvore de componentes. No lado direito os componentes selecionados podem ser posicionados e ligados, unindo a saída de um com a entrada de outro, para formar o pipeline. Na parte de baixo, um display exibe mensagens de log e é possível encontrar os botões para controlar a execução do pipeline.

A ferramenta VisPipeline possibilita a criação e execução de diferentes cenários de análise, permitindo o uso de componentes básicos, que são disponibilizados com a ferramenta, assim como o desenvolvimento de outros componentes de interesse dos usuários especialistas. Sua interface gráfica permite uma maior facilidade na construção dos pipelines 


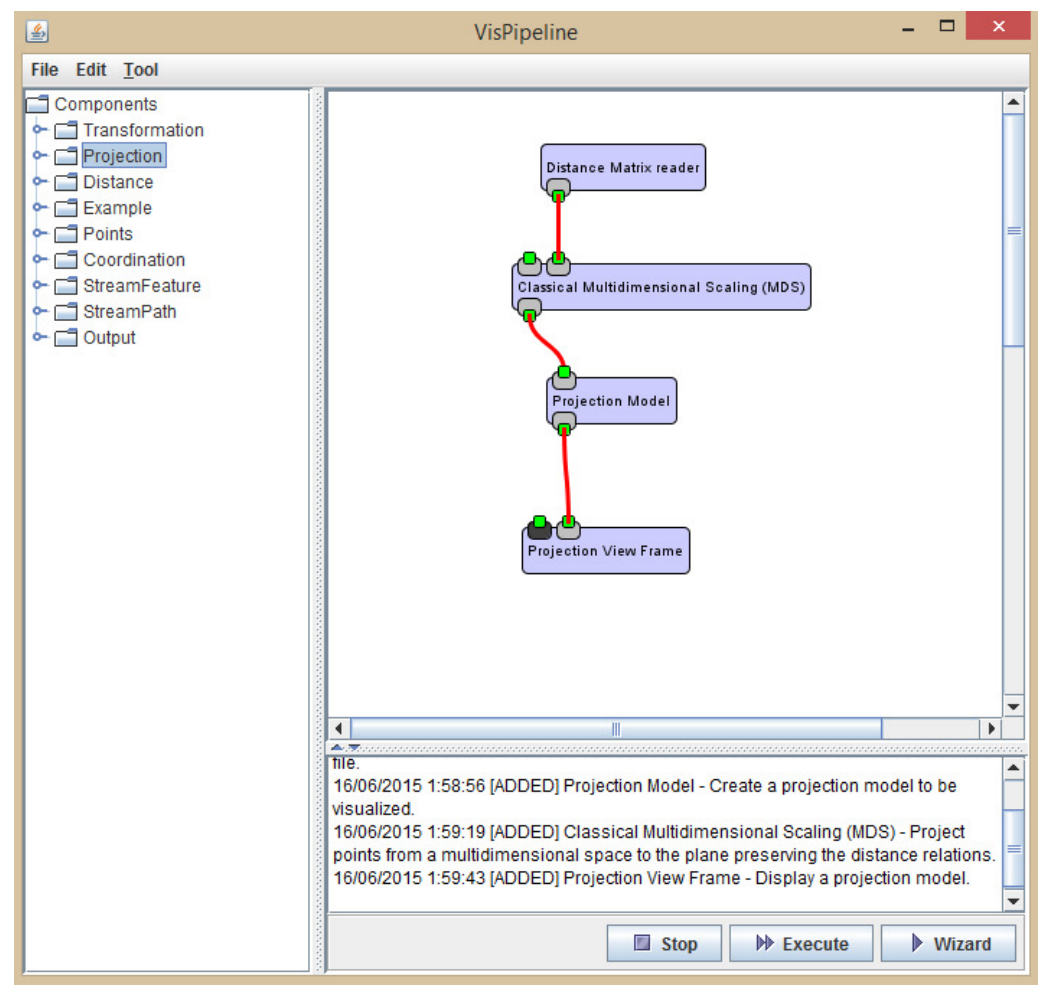

Figura 6. Interface gráfica para a construção de pipelines do VisPipeline.

de componentes, facilitando as trocas dos elementos, aumentando a interação do usuário com o processo de criação dos mapas visuais.

\section{Conclusões}

Técnicas de visualização computacional permitem extrair informações e conhecimento de grandes volumes de dados, se beneficiando da capacidade visual humana. As dificuldades associadas à gestão de dados permeiam diferentes disciplinas e domínios, tanto no cenário da pesquisa acadêmica como nos setores de serviços, industrial e agropastoril, e a visualização assume um papel importante nesse contexto.

Atualmente diferentes técnicas de visualização estão disponíveis, sendo as projeções multidimensionais técnicas que veem despertando grande interesse por sua capacidade de 
revelar estruturas de similaridade e vizinhança em conjuntos multidimensionais. Neste artigo foi apresentada uma revisão das técnicas de projeção multidimensionais e sua aplicação sobre diversos tipos de conjuntos de dados. Além de técnicas clássicas, foram apresentadas novas técnicas que buscam resolver deficiências existentes ou tratar algum tipo de dado que até então não era considerado.

Como forma de evidenciar a importância de uso de técnicas de projeção, algumas aplicações foram descritas e ilustradas. Entre as mais interessantes pode-se ressaltar a análise de coleções de documentos, de conjuntos de dados provenientes de sensores e a visualização de espaços de características. Nos trabalhos descrevendo essas aplicações é relatado que as projeções se mostram eficientes e eficazes em diversas situações, ajudando o usuário a entender, manipular e explorar os dados.

Também foram introduzidas ferramentas que estão disponíveis e permitem a realização de análises exploratórias sobre diversos conjuntos de dados. O destaque é a ferramenta VisPipeline por ser bastante flexível e permitir que os usuários possam desenvolver novos componentes para gerar novos tipos visualização.

Uma tendência da área é a junção de técnicas de análise visual com técnicas de aprendizado de máquina. Entre as contribuições que a área de aprendizado de máquina pode trazer para a visualização de informação estão: descoberta de padrões desconhecidos entre atributos, identificação de dimensões ou características irrelevantes dos dados, entre outras. Um exemplo de aplicação que pode ser melhorada por meio dessa integração é a análise de espaços de características, tarefa importante em diferentes domínios de aplicação, desde análise de imagens médicas até a recuperação de informação por conteúdo.

\section{Agradecimentos}

Os autores gostariam de agradecer à Fundação de Amparo a Pesquisa do Estado de São Paulo (FAPESP) [concessões número 2011/22749-8, 2011/17925-1 e 2014/13010-7] e ao Concelho Nacional de Desenvolvimento Científico e Tecnológico (CNPq) pelo apoio financeiro, como também a Universidade de São Paulo (USP) e a Universidade Federal de Viçosa (UFV).

\section{Referências}

[1] D. Achlioptas. Database-friendly random projections: Johnson-lindenstrauss with binary coins. J. Comput. Syst. Sci., 66(4):671-687, 2003.

[2] D. F. Andrews. Plots of high-dimensional data. Biometrics, 29:125-136, 1972. 
[3] K. Andrews, W. Kienreich, V. Sabol, J. Becker, G. Droschl, F. Kappe, M. Granitzer, P. Auer, and K. Tochtermann. The InfoSky visual explorer: exploiting hierarchical structure and document similarities. Information Visualization, 1(3/4):166-181, 2002.

[4] M. Ankerst, D. Keim, and H.-P. Kriegel. Circle segments: A technique for visually exploring large multidimensional data sets. In Proceedings of the IEEE Visualization 1996 (Vis'96), 1996.

[5] P. H. B. Aoki, P. Alessio, L. N. Furini, C. J. L. Constantino, T. T. A. T. Neves, F. V. Paulovich, M. C. F. de Oliveira, and O. N. Oliveira. Molecularly designed layer-bylayer (lbl) films to detect catechol using information visualization methods. Langmuir, 29(24):7542-7550, 2013.

[6] D. Asimov. The grand tour: a tool for viewing multidimentional data. SIAM Journal of Science \& Statistical Computing, 6:128-143, 1985.

[7] A. Becks, C. Seeling, and R. Minkenberg. Benefits of document maps for text access in knowledge management: a comparative study. In Proceedings of the 2002 ACM Symposium on Applied Computing (SAC'02), pages 621-626, New York, NY, USA, 2002. ACM.

[8] J. Beddow. Shape coding of multidimensional data on a microcomputer display. In Proceedings of the IEEE Visualization 1990 (Vis'90), pages 238-246, 1990.

[9] U. Brandes and C. Pich. Eigensolver methods for progressive multidimensional scaling of large data. In Graph Drawing, pages 42-53. Springer, 2007.

[10] M. Chalmers. A linear iteration time layout algorithm for visualising high-dimensional data. In Proceedings of the IEEE Visualization 1996 (VIS'96), pages 127-131., Los Alamitos, CA, USA, 1996. IEEE Computer Society Press.

[11] C. L. Chang and R. C. T. Lee. A heuristic relaxation method for nonlinear mapping in cluster analysis. IEEE Transactions on Systems, Man, and Cybernetics, 3:197-200, 1973.

[12] C. Chen. Information Visualization: Beyond the Horizon. Springer-Verlag New York, Inc., Secaucus, NJ, USA, 2006.

[13] Y. Chen, L. Wang, M. Dong, and J. Hua. Exemplar-based visualization of large document corpus (infovis2009-1115). IEEE Transactions on Visualization and Computer Graphics, 15(6):1161-1168, 2009.

[14] H. Chernoff. The use of faces to represent points in k-dimensional space graphically. Journal of the American Statistical Association, 68(342):361-368, 1973. 
[15] G. R. Christian, C. Blumenthal, and M. Patterson. The information explosion and the adult learner: Implication for reference librarians. The Reference Librarian, 33(6070):19-30, 2000.

[16] W. S. Cleveland. Visualizing Data. Hobart Press, Summit, New Jersey, U.S.A., 1993.

[17] T. F. Cox and M. A. A. Cox. Multidimensional Scaling. Chapman \& Hall/CRC, second edition, 2000.

[18] J. Daniels II, E. Anderson, L. Nonato, and C. Silva. Interactive vector field feature identification. IEEE Transactionson Visualization and Computer Graphics, 16(6):15601568, Nov 2010.

[19] D. M. Eler, M. Nakazaki, F. V. Paulovich, D. P. Santos, M. C. F. Oliveira, J. E. S. B. Neto, and R. Minghim. Multidimensional visualization to support analysis of image collections. In Proceedings of the XXI Brazilian Symposium on Computer Graphics and Image Processing (SIBGRAPI 2008), pages 289-296, Washington, DC, USA, 2008. IEEE Computer Society.

[20] S. G. Fadel, F. M. Fatore, F. S. Duarte, and F. V. Paulovich. Loch: A neighborhoodbased multidimensional projection technique for high-dimensional sparse spaces. Neurocomputing, 150, Part $\mathrm{B}(0): 546$ - 556, 2015.

[21] M. S. Floater. Parametrization and smooth approximation of surface triangulations. Computer Aided Geometric Design, 14(3):231-250, 1997.

[22] J. C. Gower and G. B. Dijksterhuis. Procrustes problems, volume 3. Oxford University Press Oxford, 2004.

[23] G. Grinstein, M. Trutschl, and U. Cvek. High-dimensional visualizations. In Proceedings of the 7th Data Mining Conference KDD Workshop, pages 7-19, San Francisco, CA, 2001.

[24] G. Hinton and S. Roweis. Stochastic neighbor embedding. In S. Becker, S. Thrun, and K. Obermayer, editors, Advances in Neural Information Processing Systems 15, pages 857-864. MIT Press, 2002.

[25] P. E. Hoffman. Table Visualizations: A formal model and its applications. PhD thesis, Computer Science Department, University of Massachusetts Lowell, 1999.

[26] S. Ingram, T. Munzner, and M. Olano. Glimmer: Multilevel mds on the gpu. IEEE Transactionson Visualization and Computer Graphics, 15(2):249-261, 2009.

[27] A. Inselberg. The plane with parallel coordinates. The Visual Computer - Special Issue on Computational Geometry, 1:69-91, 1985. 
[28] A. Inselberg. Multidimensional detective. In Proceedings of IEEE Symposium on Information Visualization 1997 (InfoVis'97), pages 100-107, 1997.

[29] A. Inselberg and B. Dimsdale. Parallel coordinates: a tool for visualizing multidimensional geometry. In Proceedings of the IEEE Visualization 1990 (Vis'90), pages 361-375, 1990.

[30] P. Joia, D. Coimbra, J. A. Cuminato, F. V. Paulovich, and L. G. Nonato. Local affine multidimensional projection. IEEE Transactions on Visualization and Computer Graphics, 17(12):2563-2571, Dec. 2011.

[31] F. Jourdan and G. Melancon. Multiscale hybrid mds. In IV '04: Proceedings of the Information Visualisation, Eighth International Conference on (IV'04), pages 388-393, Washington, DC, USA, 2004. IEEE Computer Society.

[32] D. A. Keim. Designing pixel-oriented visualization techniques: Theory and applications. IEEE Transactionson Visualization and Computer Graphics, 6(1):59-78, 2000.

[33] D. A. Keim and H. P. Kreigel. Visdb: Database exploration using multidimensional visualization. IEEE Computer Graphics and Applications, 14(5):40-49, September 1994.

[34] D. A. Keim and H. P. Kriegel. Visualizations techniques for mining large databases: a comparison. IEEE Transactions on Knowledge and Data Engineering, 8(6):923-936, December 1996.

[35] J.-D. Kim, H.-G. Byun, D.-J. Kim, Y.-K. Ham, W.-S. Jung, and C.-O. Yoon. A simple taste analyzing system design for visual and quantitative analysis of different tastes using multi-array chemical sensors and pattern recognition techniques. Talanta, 70(3):546 - 555, 2006.

[36] T. Kohonen. The self-organizing map. Proceedings of the IEEE, 78(9):1464-1480, 1998.

[37] S. Kullback and R. A. Leibler. On information and sufficiency. Ann. Math. Statist., 22(1):79-86, 031951.

[38] J. LeBlanc, M. O. Ward, and N. Wittels. Exploring n-dimensional databases. In Proceedings of the IEEE Visualization 1990 (Vis'90), pages 230-237, 1990.

[39] M. Martin-Merino and A. Munoz. A new sammon algorithm for sparse data visualization. In Proceedings of the 17th International Conference on Pattern Recognition (ICPR'04), pages 477-481, Washington, DC, USA, 2004. IEEE Computer Society. 
[40] S. P. Mohanty and E. Kougianos. Biosensors: a tutorial review. Potentials, IEEE, 25(2):35-40, 2006.

[41] A. Morrison, G. Ross, and M. Chalmers. A hybrid layout algorithm for sub-quadratic multidimensional scaling. In Proceedings of the IEEE Symposium on Information Visualization (InfoVis'02), pages 152-158, Washington, DC, USA, 2002. IEEE Computer Society.

[42] H. Niemann and J. Weiss. A fast-converging algorithm for nonlinear mapping of highdimensional data to a plane. IEEE Transactions on Computers, 28(2):142-147, 1979.

[43] M. C. F. Oliveira and H. Levkowitz. From visual data exploration to visual data mining: a survey. IEEE Transactions on Visualization and Computer Graphics, 9(3):378-394, 2003.

[44] V. Parra, T. Hernando, M. L. Rodríguez-Méndez, and J. A. de Saja. Electrochemical sensor array made from bisphthalocyanine modified carbon paste electrodes for discrimination of red wines. Electrochimica Acta, 49(28):5177 - 5185, 2004.

[45] F. V. Paulovich, D. M. Eler, J. Poco, C. P. Botha, R. Minghim, and L. G. Nonato. Piece wise laplacian-based projection for interactive data exploration and organization. In Computer Graphics Forum, volume 30, pages 1091-1100. Wiley Online Library, 2011.

[46] F. V. Paulovich, R. Maki, M. de Oliveira, M. Colhone, F. Santos, V. Migliaccio, P. Ciancaglini, K. Perez, R. Stabeli, A. Perinoto, O. Oliveira, and V. Zucolotto. Using multidimensional projection techniques for reaching a high distinguishing ability in biosensing. Analytical and Bioanalytical Chemistry, 400:1153-1159, 2011. 10.1007/s00216-0114853-2.

[47] F. V. Paulovich, M. L. Moraes, R. M. Maki, M. Ferreira, O. N. Oliveira Jr., and M. C. F. de Oliveira. Information visualization techniques for sensing and biosensing. Analyst, 136:1344-1350, 2011.

[48] F. V. Paulovich, L. G. Nonato, R. Minghim, and H. Levkowitz. Least square projection: a fast high precision multidimensional projection technique and its application to document mapping. IEEE Transactions on Visualization and Computer Graphics, 14(3):564-575, May/Jun 2008.

[49] F. V. Paulovich, M. C. F. Oliveira, and R. Minghim. The projection explorer: A flexible tool for projection-based multidimensional visualization. In SIBGRAPI '07: Proceedings of the XX Brazilian Symposium on Computer Graphics and Image Processing (SIBGRAPI 2007), pages 27-36, Washington, DC, USA, 2007. IEEE Computer Society. 
[50] F. V. Paulovich, R. Pinho, C. P. Botha, A. Heijs, and R. Minghim. Pex-web: Contentbased visualization of web search results. In Proceedings of the 12th International Conference on Information Visualization (IV'08), pages 208-214, Los Alamitos, CA, USA, 2008. IEEE Computer Society.

[51] F. V. Paulovich, C. T. Silva, and L. G. Nonato. Two-phase mapping for projecting massive data sets. IEEE Transactions on Visualization and Computer Graphics, 16(6):1281-1290, 2010.

[52] E. Pekalska, D. de Ridder R. P. W. Duin, and M. A. Kraaijveld. A new method of generalizing Sammon mapping with application to algorithm speed-up. In M. Boasson, J. A. Kaandorp, J. F. M. Tonino, and M. G. Vosselman, editors, Proceedings of the 5th Annual Conference of the Advanced School for Computing and Imaging (ASCI'99), pages 221-228, Delft, Netherlands, June 1999.

[53] A. C. Perinoto, R. M. Maki, M. C. Colhone, F. R. Santos, V. Migliaccio, K. R. Daghastanli, R. G. Stabeli, P. Ciancaglini, F. V. Paulovich, M. C. F. de Oliveira, O. N. Oliveira, and V. Zucolotto. Biosensors for efficient diagnosis of leishmaniasis: Innovations in bioanalytics for a neglected disease. Analytical Chemistry, 82(23):9763-9768, 2010.

[54] R. M. Pickett and G. G. Gristein. Iconographic displays fos visualizing multidimensional data. In Proceedings of IEEE Conference on Systems, Man, and Cybernetics, pages 514-519, 1988.

[55] PNNL. IN-SPIRE ${ }^{T M}$. Pacific Northwest National Laboratory (PNNL). http://inspire.pnl.gov/ (acessado em 14/09/2008), 2008.

[56] R. Rao and S. K. Card. The table lens: merging graphical and symbolic representations in an interactive focus + context visualization for tabular information. In $\mathrm{CHI}$ '94: Proceedings of the SIGCHI conference on Human factors in computing systems, pages 318-322, New York, NY, USA, 1994. ACM.

[57] A. Riul Jr., C. A. R. Dantas, C. M. Miyazaki, and O. N. Oliveira Jr. Recent advances in electronic tongues. Analyst, 135:2481-2495, 2010.

[58] S. Rose and P. C. Wong. Driftweed - a visual metaphor for interactive analysis of multivariate data. In Proceedings of IS\&T/SPIE Conference - Visual Data Exploration and Analysis, volume 3960, pages 114-121, 2000.

[59] S. T. Roweis and L. K. Saul. Nonlinear dimensionality reduction by locally linear embedding. Science, 290(5500):2323-2326, December 2000.

[60] J. W. Sammon. A nonlinear mapping for data structure analysis. IEEE Transactions on Computers, 18(5):401-409, 1969. 
[61] W. Siedlecki, K. Siedlecka, and J. Sklansky. An overview of mapping techniques for exploratory pattern analysis. Pattern Recognition, 21(5):411-429, 1988.

[62] V. D. Silva and J. B. Tenenbaum. Global versus local methods in nonlinear dimensionality reduction. In Advances in neural information processing systems, pages 705-712, 2003.

[63] V. D. Silva and J. B. Tenenbaum. Sparce multidimensional scaling using landmark points. Technical report, Standford, 2004.

[64] A. Skupin. A cartographic approach to visualizing conference abstracts. IEEE Computer Graphics and Applications, Volume 22, Issue 1:50-58, 2002.

[65] E. Tejada, R. Minghim, and L. G. Nonato. On improved projection techniques to support visual exploration of multidimensional data sets. Information Visualization, 2(4):218231, 2003.

[66] J. B. Tenenbaum, V. de Silva, and J. C. Langford. A global geometric framework for nonlinear dimensionality reduction. Science, 290(5500):2319-2323, December 2000.

[67] W. Torgerson. Multidimensional scaling of similarity. Psychometrika, 30(4):379-393, 1965.

[68] W. T. Tutte. How to draw a graph. Lodon Mathematical Society, 13:743-768, 1963.

[69] L. van der Maaten and G. Hinton. Visualizing data using t-SNE. Journal of Machine Learning Research, 9:2579-2605, 2008.

[70] J. Venna, J. Peltonen, K. Nybo, H. Aidos, and S. Kaski. Information retrieval perspective to nonlinear dimensionality reduction for data visualization. Journal of Machine Learning Research, Volume 11:451-490, 2010.

[71] J. A. Wise. The ecological approach to text visualization. Journal of the American Society for Information Science, 50(13):1224-1233, 1999. 